Purpose Little research addresses sexual pleasure in young men who have sex with men (YMSM). In this qualitative study, we developed a sexual health focused conceptual framework to explore relationships that emerged between condom use and sexual pleasure in sexual relationships among a sample of gay, bisexual, and transgender males. Methods 30 YMSM (ages 18-24 years) were recruited (through advertisements on social networking sites, participant referrals, and flyers posted at local venues frequented by YMSM) to complete a 90-minute, semi-structured interview seeking to better understand partner-seeking behaviours of YMSM. Interviews were transcribed verbatim. Analysis used inductive open coding such that emergent concepts were connected across interviews and major themes identified.

Results Median age was 22 years old $(M=21.96$; $S D=1.75)$. Most $(\mathrm{N}=18,60 \%)$ of participants self-identified as White, and gay $(\mathrm{N}=22,73 \%)$. Over $90 \%(\mathrm{~N}=28)$ reported having had sex with someone met on a dating website in the past 3 months. Five (17\%) participants reported being HIV-positive and 12 (40\%) reported a prior history of a sexually transmitted infection. Emotional effects (such as connectedness with others) and physical effects (loss of sensation and erectile difficulties) mediated the relationship between pleasure and condom use during insertive penile-anal intercourse. Specific characteristics of sexual events (e.g., use of lubricant), relationship with the partner, and of the specific sex act (including sexual position) moderated the relationship, with pleasure and satisfaction greater during receptive anal sex without a condom with emotionally intimate or regular partners.

Conclusions Our findings suggest that relationship between sexual pleasure and condom use may be mediated by both emotional and sexual factors. Prevention work with YMSM need to acknowledge the centrality of pleasure in sexual health and focus on modifiable factors that may impact pleasure among YMSM.

\section{YI.2 TEENS, THE INTERNET, AND STD RISK: FINDINGS AND LESSONS LEARNED FROM THE COMMUNICATION, HEALTH, AND TEENS (CH@T) STUDY}

doi:10.1136/sextrans-2013-051184.0079

'E R Buhi, 'E Powers, 'S Hughes, ${ }^{2} \mathrm{~N}$ Klinkenberger, ${ }^{1} \mathrm{C}$ W Wheldon, ${ }^{3} \mathrm{C}$ Rietmeijer 'University of South Florida, College of Public Health, Tampa, FL, United States, ${ }^{2}$ Physicians for Peace, Norfolk, VA, United States, ${ }^{3}$ Colorado School of Public Health, University of Colorado Denver, Denver, CO, United States

Background Few studies have examined the association between sexual health risks and online sex seeking among teenagers. The purpose of this study was to assess the associations between meeting sex partners online and a range of sexual risk behaviours and outcomes among adolescents.

Methods Participants aged 13-19 years were recruited from a publicly funded teen clinic in Florida. After obtaining informed consent/assent, 273 participants completed an audio computer-assisted self-interview (ACASI) that included questions on demographics, sexual behaviour, STD history, and online sex-seeking behaviours and experiences. Participants also provided urine samples for chlamydia and gonorrhoea testing. Data were analysed using logistic regression to identify the association between having an online sex partner and sexual behaviours/outcomes.

Results After adjusting for significant bivariate correlates, teens reporting online sex partners were more likely to be male, be multiracial, have a history of same-sex sexual activity, report a higher number of vaginal sex partners, and report a lower age at first vaginal sex. However, teens with online sex partners were no more likely to have ever had an STD or a current biological STD.

Conclusion This study is one of the first to link biological STD results to online sex-seeking data in a youth population. While meeting a sex partner online was not associated with past or current STDs, it was associated with other sexual risk behaviours. Future research is needed to examine the complex nature of online sexual partnering among adolescents and to develop intervention approaches. In-depth qualitative interviews, currently being conducted with teens, are exploring the process and context of meeting partners online. These interview data will also be discussed in light of the ACASI and biological STD data findings.

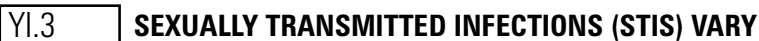 AMONG AFRICAN AMERICAN WOMEN WHO HAVE SEX WITH WOMEN BASED ON EXPOSURE TO MALE SEXUAL PARTNERS}

doi:10.1136/sextrans-2013-051184.0080

C A Muzny, H S Harbison, A M Whittington, R L Whidden, S S Richter, M G Jones, E L Austin, E W Hook. Division of Infectious Diseases, University of Alabama at Birmingham, Birmingham, UK

Introduction Little is known about partner characteristics or rates of STIs among African American women who have sex with women (AAWSW).

Methods African American women aged $\geq 16$ years attending a Health Department STD clinic were enrolled in this ongoing study if they reported sexual activity with a female partner during the preceding year. Participants completed a study questionnaire and were tested for curable (trichomoniasis, Chlamydia, gonorrhoea, and syphilis) and non-curable (HSV-2, HIV) STIs.

Results Of 128 participants reporting female partners during the preceding year, $52 \%(67 / 128)$ also reported sex with men during the same interval (WSWM). WSW and WSWM did not differ with regards to age, lifetime number of female partners, or number of female partners during the preceding year. WSWM reported increased numbers of lifetime male partners compared to WSW $(p=0.01)$. During the 30 days preceding enrollment, WSWM reported a median of 2 sexual partners (interquartile (IOR) range $0-4$ ) while WSW reported a median of 1 sexual partner (IOR 0-2). WSWM were significantly more likely than WSW to report new or casual female partners within 30 days preceding enrollment (46\% vs. $28 \% ; p=0.03$ ) while WSW were more likely to report regular female partners $(75 \%$ vs. $34 \% ; p=0.01)$. Additionally, $39 \%(26 / 67)$ of WSWM reported new or casual male partners within 30 days preceding enrollment. Although not statistically significant, diagnosis of all curable STIs (trichomoniasis, Chlamydia, gonorrhoea, and syphilis) was more common among WSWM than WSW (30\% vs. $16 \% ; p=0.07$ ). Similarly, seropositivity for HIV and HSV-2 was more than twice as common among WSWM as WSW.

Conclusions AAWSW in this study were at high risk for STIs. AAWSWM, as a subgroup, may demonstrate heightened STI rates compared to exclusive AAWSW, perhaps influenced by partnership characteristics. Sexual health services for AAWSW should take into account partner gender heterogeneity when screening for STIs.

\section{YI.4 GONOCOCCAL GENOMICS SHOWS IMPACT OF RECOMBINATION ON OBSCURING PHYLOGENETIC SIGNAL AND DISSEMINATING RESISTANCE LOCI}

doi:10.1136/sextrans-2013-051184.008

${ }^{2}$ Y Grad, ${ }^{3} \mathrm{R}$ Kirkcaldy, ${ }^{4} \mathrm{~J}$ Dordel, 'E Goldstein, ${ }^{3} \mathrm{D}$ Trees, ${ }^{3} \mathrm{H}$ Weinstock, ${ }^{4} \mathrm{~J}$ Parkhill, 'W P Hanage, ${ }^{4,5}$ S Bentley, ${ }^{1,6} \mathrm{M}$ Lipsitch. ' Center for Communicable Disease Dynamics, Department of Epidemiology, Harvard School of Public Health, Boston, MA, United States, ${ }^{2}$ Division of Infectious Diseases, Brigham and Women's Hospital, Harvard Medical School, Boston, MA, United States, ${ }^{3}$ Centers for Disease Control and Prevention, Atlanta, GA, United States, 'Wellcome Trust Sanger Institute, Hinxton, UK, ${ }^{5}$ Department of Medicine, University of Cambridge, Addenbrookes Hospital, Cambridge, UK, ${ }^{6}$ Department of Immunology and Infectious Diseases, Harvard School of Public Health, Boston, MA, United States

Background Recombination plays a significant role in the plasticity of the Neisseria gonorrhoeae genome by generating antigenic diversity and as a mechanism of spread of antibiotic resistance elements. 